\title{
MÉTODOS PARA FABRICAÇÃO DE MICROELETRODOS VISANDO A DETECÇÃO EM MICROAMBIENTES
}

\author{
Thiago R. L. C. Paixão* \\ Centro de Ciências Naturais e Humanas, Universidade Federal do ABC, Rua Santa Adélia, 166, 09210-170 Santo André - SP, Brasil \\ Mauro Bertotti \\ Instituto de Química, Universidade de São Paulo, Av. Prof. Lineu Prestes, 780, 05508-900 São Paulo - SP, Brasil \\ Recebido em 18/10/08; aceito em 28/11/08; publicado na web em 11/5/09
}

\begin{abstract}
METHODS FOR FABRICATION OF MICROELECTRODES TOWARDS DETECTION IN MICROENVIRONMENTS. Analysis at microenvironments, like single cells or in minute volumes $(\mathrm{nL})$, is an area of great interest for analytical and biological sciences. Measurements at these experimental conditions demand analytical tools (microelectrodes) capable of monitoring with rapid response, good resolution and minimal perturbation of the system. The major drawbacks in producing these microscopic electrodes have been largely overcome, principally due to the development of new fabrication methods. In this review, these procedures are described with emphasis to those devoted to the construction of microelectrodes for application in microenvironments. Examples of our efforts to use these devices as effective electrochemical sensors are also addressed.
\end{abstract}

Keywords: microelectrode; microelectrode fabrication; ensembles of microelectrodes and array of microelectrodes.

\section{INTRODUÇÃO}

\section{Definição e vantagens dos microeletrodos sobre os eletrodos de} tamanho convencional (macroeletrodos)

Microeletrodos, muitas vezes chamados de ultramicroeletrodos, são sensores químicos com dimensões micrométricas ou sub-micrométricas. ${ }^{1}$ Estes dispositivos podem ser fabricados isoladamente ou na forma de arranjos e possuem várias configurações: disco, anel, arranjo de discos, banda, cilíndrico, cônico, esfera, hemisfério, arranjo interdigitado, dentre outras.

Na definição do termo "microeletrodo", não somente fatores como tamanho e forma da superfície eletródica devem ser levados em consideração mas, adicionalmente, parâmetros temporais da técnica eletroanalítica empregada, os quais governam a espessura da camada de difusão. ${ }^{1}$ Eletrodos de tamanho convencional $\left(\mathrm{r}=10^{-3} \mathrm{~m}\right)$ podem, sob certas condições experimentais, responder como microeletrodo. Entretanto, para que esse comportamento seja mantido em outras condições experimentais é necessário que o eletrodo apresente tamanho substancialmente menor $\left(\mathrm{r}=10^{-6} \mathrm{~m}\right) .{ }^{1}$ Dessa forma, a definição de microeletrodo como eletrodo de tamanho micrométrico é arbitrária. Nesse sentido, a definição de microeletrodo pode ser mais abrangente ${ }^{1}$ e referir-se a qualquer eletrodo cuja dimensão, dentro de uma dada condição experimental, seja comparável ou menor que a da camada de difusão, $\delta$. Sob essas condições, um perfil de estado estacionário ou pseudo estado estacionário (microeletrodos cilíndricos) é obtido.

Eletrodos com a característica descrita acima possuem um comportamento eletroquímico diferenciado, proporcionando voltamogramas com perfil sigmoidal referente ao estado estacionário. A obtenção do estado estacionário deve-se predominantemente ao transporte de massa mais eficiente nessas superfícies eletródicas, em que prevalece a difusão radial.

No estado estacionário, a superfície do microeletrodo é envolvida por uma camada de difusão hemisférica. Com isso, a resposta para a alteração do potencial de um valor onde $\mathrm{j}=0$, para um valor onde $\mathrm{o}$ processo eletroquímico é controlado por difusão, pode ser descrita para um microeletrodo de disco pela Equação 1:

\footnotetext{
*e-mail: thiago.paixao@ufabc.edu.br
}

$j_{L}=\frac{n F D^{1 / 2} c}{\pi^{1 / 2} t^{1 / 2}}+\frac{4 n F D c}{\pi r}$

onde, $\mathrm{j}_{\mathrm{L}}$ é definido como densidade de corrente no estado estacionário, $\mathrm{n}$ é o número de elétrons, $\mathrm{F}$ é a constante de Faraday, $\mathrm{D}$ é o coeficiente de difusão da espécie eletroativa, c é a concentração da espécie eletroativa no seio da solução, t é o tempo após a alteração para o potencial onde o processo eletroquímico é governado por difusão e $\mathrm{r}$ corresponde ao raio do microeletrodo. O primeiro termo da equação é idêntico ao definido para um degrau de potencial para eletrodos de tamanho convencional e o segundo termo é o que define a condição de estado estacionário. Nota-se pela análise da Equação 1 que o primeiro termo é dominante em tempos curtos e o segundo, em tempos longos. Além disso, existe um valor intermediário, com relação ao tempo, onde a equação deve ser utilizada na sua forma completa. Vale salientar que essas condições de contorno na Equação 1 são dependentes do raio do microeletrodo. Dessa forma, na condição de estado estacionário a equação é descrita pela Equação 2:

$j_{L}=\frac{4 n F D c}{\pi r}$

e a corrente pela Equação 3:

$I_{L}=4 n F D c r$

onde $\mathrm{I}_{\mathrm{L}}$ é definido como corrente no estado estacionário.

Uma vez definido o conceito de microeletrodos, é necessário entender as vantagens que nas duas últimas décadas têm atraído o interesse de tantos pesquisadores da área de eletroanalítica para esta classe especial de eletrodos. Algumas propriedades únicas dos microeletrodos as quais alicerçam este interesse são: ${ }^{1-9}$ a corrente de estado estacionário para processos faradaicos é obtida em um curto espaço de tempo; a razão corrente faradaica/corrente capacitiva, $\mathrm{I}_{\mathrm{F}} / \mathrm{I}_{\mathrm{C}}$, é aumentada, uma vez que a corrente capacitiva decai proporcionalmente com a área do eletrodo, enquanto que a corrente faradaica é proporcional aos parâmetros dimensionais do eletrodo; a queda ôhmica, IR, é virtualmente desprezível devido aos baixos valores de corrente medidos com microeletrodos, 
possibilitando estudos eletroquímicos de sistemas redox em solventes com elevada resistência ou na ausência de eletrólito suporte; o potencial aplicado pode ser variado rapidamente, porque a corrente capacitiva é minimizada; a razão sinal/ruído em condições de estado estacionário é melhorada quanto comparada com eletrodos de tamanho convencional; existe aumento do transporte de massa da espécie eletroativa para a superfície do eletrodo e, conseqüentemente, pouca interferência de efeitos hidrodinâmicos sobre o sinal de corrente; o pequeno tamanho dos eletrodos pode propiciar a sua utilização para a exploração de domínios microscópicos (pequenos volumes de amostra).

A Figura 1 mostra o número de artigos publicados nas últimas décadas com base em informações do ISI Web of Science no período de 1944-2008, utilizando a palavra de busca "microelectrode". Nota-se que com o aparecimento de novas estratégias para a fabricação dos microeletrodos, por volta de 1990, a quantidade de artigos científicos em que o uso destes dispositivos é citado aumentou significativamente. Um grande número de artigos de revisão sobre microeletrodos pode ser encontrado na literatura com enfoques incidindo sobre a comparação das diferentes geometrias, descrição da teoria e vantagens e desvantagens da utilização de microeletrodos como microssensores em Química Analítica. ${ }^{16-16}$ No presente artigo pretende-se descrever os principais métodos de fabricação de microeletrodos singulares e arranjo de microeletrodos atualmente utilizados como sensores para aplicações em microambientes. A experiência de nosso grupo de pesquisa e algumas contribuições na área também serão relatadas.

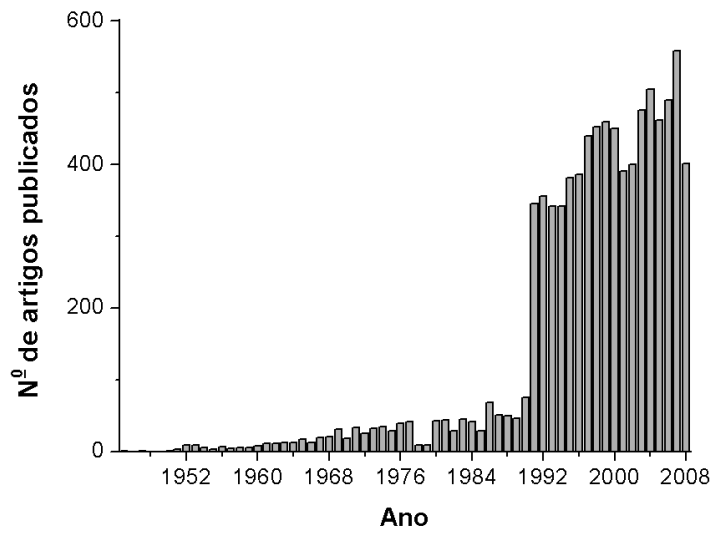

Figura 1. Número de artigos publicados desde 1944 até outubro de 2008 encontrado no banco de dados do portal ISI Web of Science com a palavra chave microelectrode

\section{MÉTODOS DE FABRICAÇÃO}

Embora ainda haja certo espaço para inovações na fabricação de microeletrodos, a grande maioria dos artigos publicados nesta área envolve a aplicação dos dispositivos em ambientes ou amostras que requerem dispositivos que possuem as características vantajosas desta classe especial de eletrodos. Esta discussão é bastante oportunamente ressaltada em recente artigo de revisão de Wightman, ${ }^{17}$ que descreve os avanços na área tomando como referência outro artigo de revisão sobre microeletrodos publicado em $1988 .{ }^{18} \mathrm{~A}$ fabricação de eletrodos micrométricos pode ser feita simplesmente selando microfibras de carbono, ou outros metais $(\mathrm{Au}, \mathrm{Cu}$ e Pt), dentro de um isolante apropriado (por exemplo, vidro). ${ }^{19-21}$ Métodos para a fabricação de eletrodos com dimensões submicrométricas são menos comuns quando comparados àqueles devotados para a construção de eletrodos micrométricos ${ }^{17}$ Todavia, em virtude dos avanços tecnológicos na área de fabricação de novos materiais de dimensões cada vez menores, esta situação tem sido revertida. ${ }^{22-24} \mathrm{~A}$ seguir, reportam-se alguns métodos de fabricação de microeletrodos de geometrias diversificadas.

\section{MICROELETRODOS DE DISCO}

\section{Microeletrodos de disco fabricados por encapsulamento em vidro ou com resina}

Microeletrodos de disco são usualmente construídos por meio de encapsulamento da fibra em vidro ou resina epóxi. ${ }^{5,25-29}$ Nos procedimentos de encapsulamento em vidro, ${ }^{30}$ utiliza-se um capilar e uma das extremidades é fechada por aquecimento. Após esta etapa, uma fibra de diâmetro nominal de $10-50 \mu \mathrm{m}$ é posicionada no interior da extremidade selada do capilar, e a outra extremidade do capilar é conectada a uma bomba de vácuo. A extremidade selada do capilar, em cujo interior se encontra a fibra, é então aquecida utilizando-se uma resistência ou a chama de um bico de Bunsen. Dessa forma, o vidro é selado sobre a microfibra sem a exposição da microfibra às altas temperaturas. Após esse procedimento, efetua-se o contato externo da microfibra com um fio de $\mathrm{Cu}$ ou $\mathrm{Ni} / \mathrm{Cr}$ utilizando uma resina epóxi condutora, mercúrio ou cola de prata. Realizado o contato elétrico, a extremidade inicialmente selada é quebrada ou polida a fim de expor a superfície da fibra.

Esse procedimento é bastante usual e funciona relativamente bem ao serem empregadas microfibras de platina e ouro com raio de até $5 \mu \mathrm{m}$. Entretanto, a construção de eletrodos de dimensões menores (por volta de $1 \mu \mathrm{m}$ ) exige o uso de outro tipo de microfibra, chamada Wollaston (Goodfellow Metals, Cambridge, UK). Estas microfibras de ouro ou platina são adquiridas comercialmente e vêm recobertas por uma camada de prata $(50-100 \mu \mathrm{m})$ para facilitar o manuseio a olho nu. A fabricação de microeletrodos utilizando esse tipo de fibra ${ }^{19-21}$ envolve a inserção de aproximadamente $2 \mathrm{~cm}$ dessa fibra em um capilar de vidro de aproximadamente $1 \mathrm{~mm}$. Vale destacar que, nesse procedimento, a extremidade do capilar onde está situada a fibra não é fechada, como reportado anteriormente na fabricação de microeletrodos com fibras convencionais. A extremidade do vidro que contém a fibra é mergulhada em uma solução contendo $50 \%$ (v/v) de $\mathrm{HNO}_{3}$ para dissolver a camada de prata. A quantidade de ácido nítrico que entra no capilar de vidro por capilaridade é controlada realizando-se uma pressão positiva na extremidade oposta do capilar. Dessa forma, somente cerca de 4-8 mm da fibra Wollaston são expostos à solução de $\mathrm{HNO}_{3}$. Após esta etapa, lava-se bem o conjunto com água destilada para remoção do ácido nítrico excedente. A água excedente é removida por aquecimento por pelo menos $2 \mathrm{~h}$. Em procedimento final, o vidro é selado sobre a parte exposta da fibra empregando-se o método já descrito anteriormente.

Resultados melhores no processo de selagem podem ser alcançados utilizando equipamentos apropriados para trabalhos com capilares de vidro, os pipette puller (puxador de pipeta). Estes instrumentos funcionam à base de aquecimento do capilar de vidro com resistências elétricas ou por laser ${ }^{31-33}$. Este último método possui vantagens, pois permite um controle mais apurado do aquecimento. Entretanto, tratase de equipamentos de custo mais elevado do que o empregado para aquecimento elétrico. Nesse procedimento (Figura 2) de selagem, a microfibra é posicionada no centro de um capilar de vidro ao qual se pode aplicar vácuo para minimizar problemas posteriores oriundos de eventual presença de bolhas. Após a fixação do conjunto capilar/ microfibra no instrumento, um programa de aquecimento controlado é elaborado em função do tipo de fibra e vidro utilizados. Vale salientar que o aquecimento é aplicado a apenas uma fração do conjunto (fibra/ vidro). Normalmente nesse tipo de processo de selagem, um primeiro programa de aquecimento é aplicado para selar o vidro na microfibra e um segundo programa de aquecimento, para esticar o vidro sobre a 
microfibra. Desta forma, a espessura da parede do isolante (vidro) é diminuída e abrem-se perspectivas para a miniaturização dos dispositivos fabricados. Neste sentido, cumpre ressaltar que microfibras do tipo annealed, mais moles do que as convencionais, podem ser afinadas utilizando-se um pipette puller. ${ }^{33}$ Nestas condições, microeletrodos de dimensões submicrométricas podem ser fabricados partindo-se de fibras de dimensões convencionais (com algumas dezenas de micrometros). Após a etapa 3 da Figura 2 pode ser realizado um corte do conjunto vidro e microfibra para exposição da área eletroativa. A possibilidade da construção de eletrodos de raios menores que o da microfibra de partida é interessante, já que um dos principais empecilhos do processo de fabricação de eletrodos com raios menores que $10 \mu \mathrm{m}$ é a manipulação da microfibra, muito dificultada sem a utilização de um microscópio.

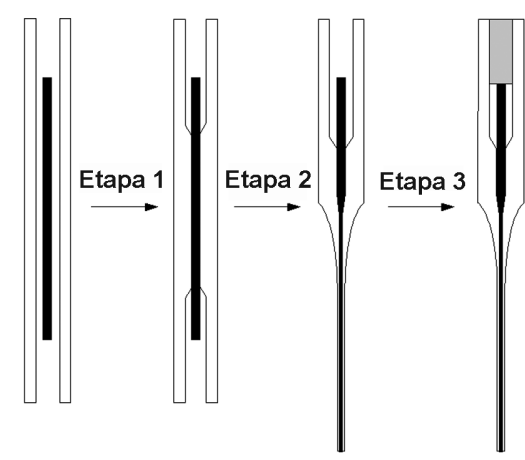

Figura 2. Esquema do processo de fabricação de um microeletrodo de disco utilizando um pipette puller. Etapa 1: processo térmico de selagem do vidro na fibra; etapa 2: processo de térmico e mecânico para afinamento do conjunto vidro e microfibra e etapa 3: contato elétrico externo

\section{Microeletrodos de disco fabricados por ablação a laser}

Alternativamente, microeletrodos de disco também podem ser fabricados por ablação a laser ${ }^{34}$ (processo de remoção de material de uma superfície sólida por irradiação com um feixe de laser) de uma camada polimérica, criando-se furos da ordem de 7-38 $\mu \mathrm{m}$, com uma precisão de $1 \mu \mathrm{m}$. No processo descrito na literatura, ${ }^{34}$ esses microorifícios foram preenchidos com tinta condutora de carbono após o processo de ablação. O contato elétrico foi feito pela deposição de um filme metálico, depositado anteriormente, em uma das faces da camada polimérica.

\section{Microeletrodos de disco fabricados a partir da deposição química em fase vapor}

Microeletrodos de disco podem também ser fabricados pela técnica de deposição química em fase vapor, ${ }^{35-41}$ processo no qual a microfibra é isolada com uma fina camada de sílica. A formação do filme ocorre pela deposição da sílica a partir dos seus precursores gasosos $\left(\mathrm{SiCl}_{4}, \mathrm{H}_{2}\right.$ e $\left.\mathrm{O}_{2}\right)$ ou pela deposição seqüencial de $\mathrm{Si}(\mathrm{OEt})_{4} \mathrm{e}$ dos precursores gasosos. Neste segundo caso, a adesão do filme de sílica sobre as microfibras é mais efetiva, enquanto no procedimento em que se empregam os precursores gasosos $\left(\mathrm{SiCl}_{4}, \mathrm{H}_{2}\right.$ e $\left.\mathrm{O}_{2}\right)$ filmes mais finos são obtidos. Microeletrodos de disco da ordem de 5 a 10 $\mu \mathrm{m}$ podem ser obtidos por esse método. ${ }^{35-37}$

Esse procedimento de fabricação apresenta uma série de vantagens: eletrodos de diferentes materiais e tamanhos podem ser fabricados e modificados alterando-se os precursores e os parâmetros experimentais; o filme isolante é fabricado sobre a própria superfície da microfibra propiciando excelente adesão e selagem; a deposição química em fase vapor pode possibilitar um filme mais uniforme quando comparado com outras técnicas de deposição. Um exemplo da flexibilidade desse método é a fabricação de microeletrodos constituídos por nanotubos de carbono fabricados a partir da deposição química em fase vapor utilizando uma atmosfera de acetileno. ${ }^{42}$

\section{Microeletrodos de disco constituídos de esferas de ouro}

Miles e colaboradores, ${ }^{43}$ usando esferas de ouro com diâmetro ao redor de 1,5-3,0 $\mu \mathrm{m}$, construíram microeletrodos de disco de ouro com diâmetro total (superfície eletródica + vidro) $\leq 5 \mu \mathrm{m}$. Os autores utilizaram como suporte para as esferas de ouro uma pipeta de vidro afinada utilizando um pipette puller. O capilar de vidro foi, então, preenchido com a suspensão de esferas de ouro. Em seguida, um programa de aquecimento utilizando o puller foi empregado para evaporar a água (120 $\left.{ }^{\circ} \mathrm{C} / 1 \mathrm{~h}\right)$ e para sinterizar as esferas de ouro $\left(580{ }^{\circ} \mathrm{C} / 2 \mathrm{~h}\right)$. Para aumentar a eficiência do processo de selagem das esferas no vidro, uma etapa adicional usando resina epóxi foi utilizada $\left(120^{\circ} \mathrm{C} / 3 \mathrm{~h}\right.$, em um forno). Realizado o processo de selagem, o contato elétrico foi estabelecido entre as pérolas e um fio metálico, utilizando uma solução eletrolítica e, quando necessário, o eletrodo foi polido cuidadosamente. Vale destacar que esse processo apresenta um baixo custo quando comparado àquele em que se utilizam microfibras comerciais, viabilizando a fabricação de microeletrodos descartáveis. Além disso, dispositivos com áreas menores podem ser obtidos, pois se trabalha com nanopartículas de ouro. Finalmente, cumpre ressaltar que este procedimento é mais simples, pois o manuseio de fibras de dimensões reduzidas é dispensado.

De maneira parecida, a literatura reporta o uso de esferas de irídio como alternativa às de ouro. ${ }^{44}$ Entretanto, após a fabricação do microeletrodo um tratamento eletroquímico $\left(\mathrm{H}_{2} \mathrm{SO}_{4} 0,5 \mathrm{~mol} \mathrm{~L}^{-1}\right.$, variação de potencial de $-0,25$ até $1,25 \mathrm{~V}$, com uma velocidade de varredura de 3 $\mathrm{V} \mathrm{s}^{-1}$ ) foi realizado para a formação de um filme de $\mathrm{IrO}_{\mathrm{x}}$. Esse eletrodo modificado foi utilizado como um sensor potenciométrico de $\mathrm{pH}$.

\section{MICROELETRODOS CILÍNDRICOS E CÔNICOS}

Como mostrado anteriormente, processos de fabricação de microeletrodos podem resultar em um afinamento do diâmetro da microfibra de partida, resultando em eletrodos de dimensões menores que a da microfibra de partida. A utilização de $\mathrm{HF}^{32,45}$ para a remoção do vidro após a etapa 2 da Figura 2 pode propiciar a construção de microeletrodos cônicos e esse tipo de estratégia vem sendo cada vez mais utilizado para a fabricação de microeletrodos estruturalmente microscópicos. Tal fato se prende à possibilidade de uso destes dispositivos em microambientes como, por exemplo, em células singulares para a caracterização de diversos processos biológicos. ${ }^{46-52}$ Nosso grupo de pesquisa desenvolveu recentemente método para o monitoramento de ascorbato em neuroblastomas, ${ }^{52}$ utilizando microeletrodos de fibra de carbono desgastados eletroquimicamente. Estas células podem conter o gene responsável por uma doença neurodegenerativa (ELA, Esclerose lateral amiotrófica) e, a partir dos resultados obtidos, foi possível verificar maior consumo de ascorbato por células portadoras da doença. Os resultados foram coerentes com os níveis de estresse oxidativo induzido pela doença, indicando que o ascorbato atua no mecanismo de defesa dessas células. Nesse sentido, métodos para reduzir as dimensões de microfibras de partida vêm sendo reportados na literatura e os procedimentos mais amplamente utilizados envolvem desgaste eletroquímico ou utilização de chama rica em oxigênio. Com base nestes procedimentos, tem sido possível obter eletrodos cuja área eletroativa é da ordem de nanômetros.

O desgaste de fibras de carbono por aquecimento é prática relativamente antiga e o tamanho mínimo das pontas obtidas é menor 
que $1 \mu \mathrm{m} .{ }^{53,54}$ As microfibras assim preparadas são posteriormente fixadas ao vidro utilizando-se resina epóxi e a parte não exposta da fibra é submetida à chama para posterior desgaste. Com isso, o processo de desgaste da superfície é dificultado pela resina, resultado em superfícies mais irregulares. Esse aspecto da superfície eletródica influenciava significativamente no comportamento eletroquímico do microeletrodo.

Tentando solucionar esse problema, procedimento baseado no desgaste da microfibra utilizando chama, sem a necessidade de uso de resina epóxi antes do processo de desgaste, foi desenvolvido. ${ }^{55}$ Além disso, métodos utilizando desgaste por um feixe de íons de argônio também foram reportados como alternativa ao processo de fabricação por chama. ${ }^{56}$ Vale ressaltar que as superfícies obtidas por esses procedimentos, quando comparadas com as anteriores, apresentam uma superfície mais homogênea.

Como alternativa aos métodos por desgaste com aquecimento surgiram estratégias baseadas no desgaste eletroquímico das microfibras. A construção de eletrodos hemisféricos ou cônicos de raios inferiores a $1 \mu \mathrm{m}$ por técnicas de corrosão eletroquímica foi primeiramente descrita por Penner e colaboradores. ${ }^{57}$ Esse procedimento envolve a corrosão de microfibras até a formação de uma ponta afinada ou um cone e foi adaptado de métodos utilizados para a fabricação de pontas de prova para microscopia de tunelamento. ${ }^{58}$ Penner e colaboradores $^{57}$ desgastaram eletroquimicamente microfibras de Pt e PtIr até a formação de pontas afinadas. O processo de desgaste eletroquímico foi realizado em uma solução aquosa contendo $\mathrm{NaOH} 2 \mathrm{~mol} \mathrm{~L}^{-1} \mathrm{e}$ $\mathrm{KCN} 6 \mathrm{~mol} \mathrm{~L}^{-1}$. Um potencial de $25 \mathrm{~V}$ AC entre a microfibra e um contra eletrodo de grafite foi aplicado e, em seguida, a microfibra afinada foi selada em vidro. Este trabalho pioneiro deu origem a inúmeras alternativas para a fabricação de microeletrodos à base de corrosão eletroquímica; a Tabela 1 fornece parâmetros relevantes destes procedimentos.

Nosso grupo de pesquisa desenvolveu metodologia para o afinamento de fibras de platina de diâmetro inicial igual a $100 \mu \mathrm{m}$, empregando-se desgaste eletroquímico com um sistema bifásico. Este consiste em solução saturada de $\mathrm{CaCl}_{2} /$ acetona (1:1 em volume) $(3 \mathrm{~mL})$ e $\mathrm{CCl}_{4}(3 \mathrm{~mL})$ e foi adaptado de processos para a fabricação de pontas de prova para microscopia de tunelamento. ${ }^{71} \mathrm{~A}$ acetona foi utilizada, pois solventes orgânicos solúveis em água provocam uma diminuição do tamanho das bolhas. Desta maneira, pode-se evitar um desgaste irregular da superfície da fibra pela adsorção de bolhas maiores. ${ }^{72}$

No método proposto, utilizou-se um sistema que consiste em dois eletrodos para o desgaste eletroquímico conforme apresentado na Figura 3. A microfibra de platina e os eletrodos de grafite foram obtidos comercialmente. Dois eletrodos de grafite foram utilizados de maneira a se obter uma distribuição mais uniforme do potencial aplicado entre os eletrodos de grafite e a microfibra de platina, evitando assim uma corrosão irregular da fibra. Procedimento similar reportado na literatura ${ }^{73}$ utiliza dois eletrodos de platina no processo, mas optou-se pelo grafite pois há desgaste de material eletródico durante o processo de corrosão da microfibra de platina.

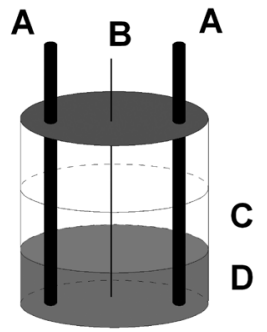

Figura 3. Esquema da célula eletroquímica utilizada para o desgaste eletroquímico. Eletrodos de grafite (A), fibra de platina $(B)$, solução para a corrosão: $\mathrm{CaCl}_{2}$ saturada $(\mathrm{C})$ e solução não condutora: $\mathrm{CCl}_{4}(\mathrm{D})$

Os eletrodos foram conectados a um gerador AC $(60 \mathrm{~Hz})$ (custo $\mathrm{R} \$ 5,00$ a 10,00 ) de valor eficaz igual a $5 \mathrm{~V}$, dispensando-se o uso de equipamentos mais caros e sofisticados (programador universal acoplado a um potenciostato-galvanostato ou geradores de função ${ }^{70}$ ). O final do processo de corrosão ocorre quando a fibra se rompe na solução de $\mathrm{CaCl}_{2}$ e acetona (15-16 min após o inicio do processo), formando-se então duas extremidades "apontadas", ao invés de somente uma como nos procedimentos anteriormente descritos.

A utilização de um potencial $\mathrm{AC}$, ao contrário de um potencial DC, é importante porque o processo de corrosão da platina não é muito eficiente. O potencial alternado reverte o processo de passivação da superfície, pois na fase negativa de potencial a camada de óxido é dis-

Tabela 1. Microeletrodos fabricados por procedimentos de desgaste eletroquímico

\begin{tabular}{|c|c|c|c|}
\hline Material & Método de fabricação & Raio efetivo/nm & Ref. \\
\hline Fibra de carbono & $\mathrm{K}_{2} \mathrm{Cr}_{2} \mathrm{O}_{7} 0,5 \mathrm{~mol} \mathrm{~L}^{-1} \mathrm{e} \mathrm{H}_{2} \mathrm{SO}_{4} 5 \mathrm{~mol} \mathrm{~L}^{-1}, 6-15 \mathrm{~V} \mathrm{AC}$ & $>3500$ & 59 \\
\hline Fibra de carbono & $\mathrm{NaOH} 0,01 \mathrm{~mol} \mathrm{~L}^{-1}, 4-5 \mathrm{~V}$ AC & 500 e $>42$ & 60,61 \\
\hline Fibra de carbono & $\begin{array}{c}\mathrm{NaCl} 0,9 \% \text { (correntes de } 0,3-4,5 \mathrm{~mA} \text {, frequência do pulso } 10 \mathrm{~Hz} \text {, } \\
\text { duração do pulso } 1-5 \mathrm{~ms} \text { ) }\end{array}$ & $2000-4000$ & 62 \\
\hline $\mathrm{Pt}$ & Solução saturada de $\mathrm{NaNO}_{3}, 1,2 \mathrm{~V}$ AC & $13,49,120$ e 1200 & 63 \\
\hline PtIr & $\mathrm{NaOH} 2 \mathrm{~mol} \mathrm{~L}^{-1}$ e KCN 6 mol L-1 & $>1,6$ & 57 \\
\hline PtIr & $\mathrm{CaCl}_{2}, \mathrm{HCl}, \mathrm{H}_{2} \mathrm{O}, 25 \mathrm{~V}$ AC & Alguns nm & 64 \\
\hline $\mathrm{Pt}$ & $\mathrm{NaCN} 6 \mathrm{~mol} \mathrm{~L}^{-1}$ ou $\mathrm{CaCl}_{2}(50 \%$ v/v em água), $5 \mathrm{~V} \mathrm{AC}$ & $2-150 \mathrm{~nm}$ & 65 \\
\hline $\mathrm{Pt}$ & $\mathrm{CaCl}_{2}(50 \% \mathrm{v} / \mathrm{v}), \mathrm{HCl}(25 \% \mathrm{v} / \mathrm{v})$ e $\mathrm{H}_{2} \mathrm{O}(25 \% \mathrm{v} / \mathrm{v}), 2 \mathrm{~V} \mathrm{AC}$ & Alguns nm & 66 \\
\hline $\mathrm{Pt}$ & $\mathrm{NaNO}_{2} 10 \mathrm{~mol} \mathrm{~L}^{-1}, 1,6 \mathrm{~V} \mathrm{AC}$ & 1,3 & 67 \\
\hline $\mathrm{Ag}$ & $\mathrm{NH}_{4} \mathrm{OH}$ (35\%) diluído 6:1 em água, 4,0 V DC & 50,70 e 2600 & 68 \\
\hline $\mathrm{Au}$ & $\mathrm{HCl} 3,6 \mathrm{~mol} \mathrm{~L}^{-1}, 1,7 \mathrm{~V} \mathrm{DC}$ & $50-250$ & 69 \\
\hline \multirow[t]{2}{*}{$\mathrm{Au}$ e $\mathrm{Pt}$} & $\mathrm{NaCN} 6 \mathrm{~mol} \mathrm{~L}^{-1}$ e $\mathrm{NaOH}$ 0,1 mol & $>10$ & $70 *$ \\
\hline & $\mathrm{L}^{-1}, 4 \mathrm{~V} \mathrm{AC}$ & & \\
\hline
\end{tabular}

\footnotetext{
* microeletrodo de disco
} 
solvida. Desta maneira, a limpeza da superfície do eletrodo é efetuada para posterior oxidação na fase positiva de potencial. A Figura 4 mostra um esquema do processo de corrosão durante a microfabricação do microeletrodo de platina. Na Figura 5 é mostrada a microscopia eletrônica de varredura de uma microfibra de platina desgastada de acordo com o procedimento descrito. Microeletrodos com essa geometria foram utilizados por nosso grupo para o mapeamento da concentração de ácido ascórbico em laranjas e, a partir desses resultados, correlações entre a concentração de ácido ascórbico e o estágio de maturação da fruta foram realizadas sem a necessidade da extração do suco da laranja. ${ }^{74}$

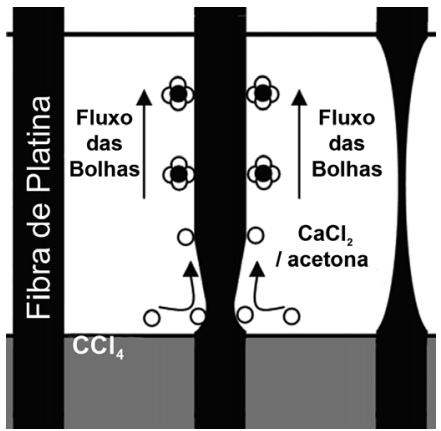

Figura 4. Esquema do processo de desgaste eletroquímico da fibra de platina em função do tempo (t). $\mathrm{Pt}^{4+}=\bullet \mathrm{Cl}^{-}=\mathrm{o}$

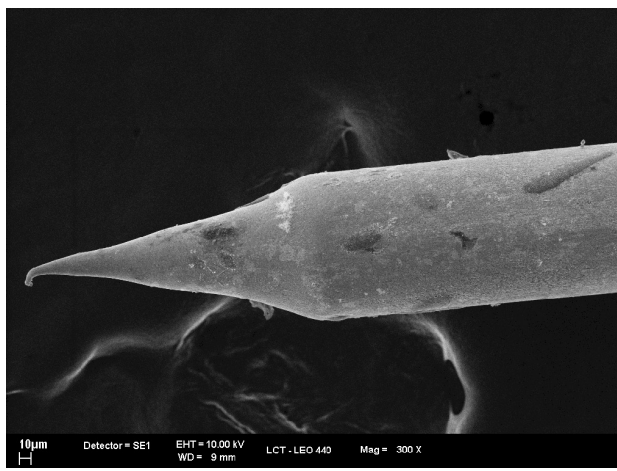

Figura 5. Microscopia eletrônica de varredura de fibra de platina que sofreu um processo de desgaste eletroquímico utilizando-se um transformador de potencial eficaz de $5 \mathrm{~V}$

Após o processo de desgaste eletroquímico da microfibra é necessário isolá-la para a definição da área eletroativa. Com o intuito de fabricar dispositivos estruturalmente microscópicos (isto é, o conjunto fibra + suporte possui dimensões micrométricas), a fibra deve ser revestida com isolante apropriado para a posterior utilização em microambientes. $\mathrm{Na}$ literatura reportam-se vários procedimentos para o revestimento das microfibras e, entre estes, pode-se citar a pulverização catódica (sputtering) de um material isolante ${ }^{75}$ e o uso de capilares afinados ${ }^{33}$ (utilizando um pipette puller), vernizes, ${ }^{76,77}$ esmalte de unha, ${ }^{69}$ parafina fundida, ${ }^{78}$ pérolas de vidro fundido ${ }^{57,79}$ e cera específicas. ${ }^{58,64}$ Processos de deposição eletroquímica empregando tintas eletroforéticas comerciais

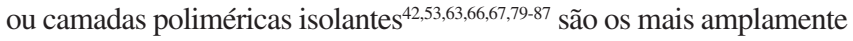
utilizados e resultam em camadas de isolante da ordem de nanômetros. Tintas eletrofóreticas anódicas apresentam em sua composição ácido poliacrílico (PAAH), que em excesso de base se desprotona formando a espécie PAA: Com a aplicação de um programa de potencial apropriado entre dois eletrodos (microfibra + eletrodo de grafite ou um fio de platina em expiral), pode-se diminuir o $\mathrm{pH}$ em região vizinha à da superfície da microfibra e, com isso, a espécie PAA é protonada. Sendo a espécie PAAH insolúvel em água, deposita-se sobre a microfibra. Em seguida, um processo de cura da película por temperatura faz com que a mesma encolha expondo, assim, a ponta afinada da microfibra. Processos subseqüentes podem ser utilizados para reparar pequenos defeitos de homogeneidade do processo de isolamento. Outros métodos, como polimento ou corte de um pedaço do microfibra isolada, ${ }^{35,37,53,75,88-93}$ descarga aplicada por um campo elétrico, ${ }^{92}$ utilização de um feixe de elétrons ${ }^{94}$ ou íons ${ }^{95} \mathrm{e}$ microscopia de tunelamento,${ }^{55}$ são também reportados na literatura para remover a camada de isolante da ponta da microfibra afinada. Além disso, a simples polarização em potenciais negativos da microfibra em meio ácido, resultando na evolução de hidrogênio, pode auxiliar na exposição de microfibras afinadas e isoladas com polifenol. ${ }^{42}$

Com o objetivo de desenvolver nova estratégia para isolar as microfibras afinadas, utilizou-se um plastificante de borracha (PlastiFilm, Quimatec $\left.{ }^{\circledR}\right)$. A microfibra foi mergulhada no plastificante e, em seguida, a ponta afinada foi direcionada para cima para que houvesse escorrimento do plastificante, conforme mostrado na Figura 6. A Figura 7 apresenta voltamogramas cíclicos registrados com microfibras de ouro e platina isoladas empregando-se o procedimento proposto. Os perfis voltamétricos (sigmoidais) típicos obtidos com estes microeletrodos indicam a prevalência do processo de difusão radial e atestam a validade da estratégia proposta para a fabricação de dispositivos estruturalmente microscópicos.

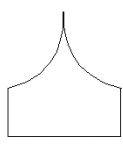

(A)

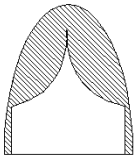

(B)

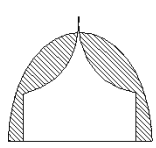

(C)
Figura 6. Esquema do processo de fabricação dos microeletrodos após desgaste eletroquímico: (A) fibra com ponta afiada, (B) imersão da fibra no plastificante e $(C)$ processo de secagem do plastificante de borracha

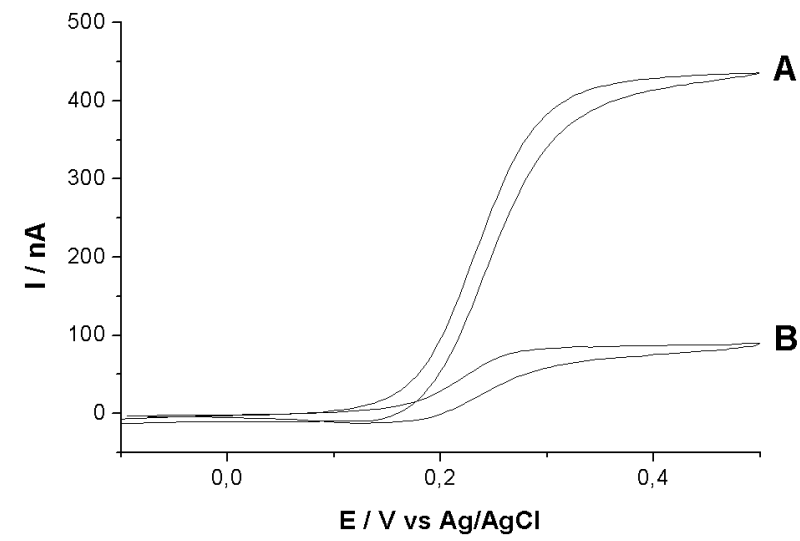

Figura 7. Voltamogramas cíclicos registrados em solução de $\mathrm{K}_{4} \mathrm{Fe}(\mathrm{CN})_{6} 20$ mol $L^{-1}+K C l 0,1 \mathrm{~mol} \mathrm{~L}^{-1}$. A e $B$ referem-se a dois diferentes microeletrodos de platina (A) e ouro (B) fabricados pelo processo de desgaste eletroquímico. Velocidade de varredura: $50 \mathrm{mV} \mathrm{s}^{-1}$

\section{MICROELETRODOS DE ANEL}

Ewing e colaboradores ${ }^{96,97}$ desenvolveram metodologia para a fabricação de um eletrodo de carbono com formato de anel, a partir da pirólise do metano dentro de capilares de quartzo afinados por um pipette puller. Anéis com diâmetro da ordem de 0,5 até $3,9 \mu \mathrm{m}$ foram obtidos com base nesse processo de fabricação. Vale salientar que o tamanho total do eletrodo, considerando a camada de vidro, foi da ordem de 1-4 $\mu \mathrm{m}$. Após a fabricação do anel nas paredes do capilar de quartzo, este era preenchido com uma resina epóxi para preenchimento interno do anel de carbono fabricado na parede do quartzo. Finalizado 
o processo de cura da resina, o vidro era quebrado, expondo o anel de carbono formado pelo processo de pirólise do metano. Como vantagens associadas a este processo podem ser citados o baixo custo e a alta eficiência do processo de fabricação dos microeletrodos.

Microeletrodo de anel são particularmente atrativos levando-se em conta o uso de fibras ópticas em experimentos fotoeletroquímicos ${ }^{88,98} \mathrm{e}$ no acoplamento de técnicas ${ }^{90}$ eletroquímicas e fotoquímicas (microscópio fotoeletroquímico). Nesses procedimentos, um microeletrodo de anel é fabricado dentro de uma fibra óptica de ouro comercial ou de uma fibra óptica cujas paredes internas são recobertas com ouro. No interior da fibra óptica é efetuado o revestimento com um material isolante (resina epóxi ou outro polímero não condutor) por dip-coating. Como resultado, essa fibra óptica passa a servir não somente como um probe para a fonte de luz, mas também como um microeletrodo com diâmetro interno da ordem de 2-400 $\mu \mathrm{m}$ e espessura do anel de 2-35 $\mu \mathrm{m}$.

Atualmente, o acoplamento de técnicas como microscopia eletroquímica e NSOM (Near field Scanning Optical Microscopy) também vem sendo amplamente aproveitado..$^{80,81}$ Nestes casos, existe uma etapa adicional que envolve o afinamento da fibra óptica usando um pipette puller.$^{81,99,100}$ Microeletrodos fabricados por essas técnicas possuem anel com diâmetro interno menor que $1 \mu \mathrm{m}$.

\section{MICROELETRODOS DE ESFERA}

A disponibilidade de microesferas de ouro (de diâmetro aproximado igual a $8 \mathrm{~nm}$ ) permitiu o desenvolvimento de método no qual as partículas são empacotadas em capilares de vidro afinados cujas paredes internas são revestidas por uma camada de carbono, ${ }^{101}$ a partir da pirólise de metano. Uma solução de ditióis, contida no interior do capilar, foi responsável pela formação das monocamadas de ouro via interação química, após a etapa de inserção do conjunto anterior em solução contendo as esferas de ouro. Obtém-se, desta forma, esferas micrométricas na ponta do capilar, as quais são compostas por nanopartículas agregadas em razão da presença dos grupamentos $\mathrm{SH}$ dos ditióis. O tamanho das microesferas pode ser controlado com base no diâmetro do orifício do capilar de vidro ou na concentração da solução de ditiol (responsável pelo agrupamento das partículas de ouro). Microeletrodos fabricados por este método possuem por volta de 1 a $30 \mu \mathrm{m}$. Como vantagens, esse método de fabricação permite a construção de eletrodos de maneira reprodutível, simples e controlada.

\section{MICROELETRODOS HEMISFÉRICOS}

$\mathrm{Na}$ literatura reporta-se que a interpretação de resultados obtidos no estado-estacionário, para microeletrodos de disco, pode ser complicada em alguns casos porque a corrente de estado estacionário próxima da borda do microdisco apresenta valores maiores que no centro. ${ }^{102-112}$ Dessa forma, a análise matemática dos resultados obtidos nestes casos é dificultada. ${ }^{104,107-112}$ Microeletrodos com geometria hemisférica não apresentam esse problema, uma vez que a densidade de corrente no estado estacionário é uniforme em toda a superfície do eletrodo. Além disso, microeletrodos não planares sofrem menos problemas de bloqueio do isolante resultantes de imprecisões no processo de isolamento da microfibra. ${ }^{113}$

Microeletrodos com este tipo de geometria são usualmente fabricados pela redução eletroquímica de $\mathrm{Hg}_{2}{ }^{2+}$ sobre a superfície de microeletrodos de disco. ${ }^{26,114,115} \mathrm{O}$ crescimento da camada de mercúrio pode ser acompanhado em função do tempo, utilizando-se um microscópio óptico imerso na solução de deposição, conforme reportado na literatura. ${ }^{26}$ Processos de fabricação de eletrodos com essa geometria utilizando desgaste eletroquímico e isolamento com material polimérico para microfibras de Pt-It também foram reportados na literatura. ${ }^{79}$

\section{MICROELETRODOS DE BANDAS}

Microeletrodos de banda possuem somente uma das dimensões em escala microscópica, mas as características difusionais associadas ao elevado transporte de massa são preservadas e conferem aos voltamogramas o clássico comportamento sigmoidal. Levando em consideração que as outras duas dimensões não são microscópicas, os valores de corrente obtidos com eletrodos que apresentam esta geometria são relativamente elevados. Uma característica importante de microeletrodos de banda diz respeito ao fato de não serem atingidas situações de estado estacionário em tempos curtos, como no caso das microesferas e dos microdiscos. Um dos primeiros procedimentos empregados para a fabricação de microeletrodos de banda consistiu no encapsulamento da placas finas de ouro e platina (espessura 20$500 \AA ̊$ e 0,5 e 1,0 cm de comprimento e largura, respectivamente) preparadas por pulverização catódica. Após procedimento de polimento adequado, superfície constituída por uma banda de espessura definida foi exposta e obteve-se voltamograma com perfil sigmoidal característico de um microeletrodo. ${ }^{116}$ Outros procedimentos para fabricação desse tipo de microeletrodo baseiam-se na imobilização de películas de materiais condutores em vidro fundido ${ }^{117}$ e entre placas isolantes. ${ }^{118}$ Baseado no procedimento de isolamento de películas metálicas, microeletrodos de banda também foram fabricados utilizando a impressão de tintas condutivas entre substratos não condutores. ${ }^{119-121}$ Além disso, técnicas litográficas, ${ }^{122}$ por ablação a laser ${ }^{123}$ e screenprinted $^{121,124}$ também estão citadas na literatura para fabricação de microeletrodos com essa geometria. Entretanto, devido a somente uma das dimensões dos eletrodos apresentar tamanho micrométrico ou submicrométrico, a aplicação desse tipo de geometria para medidas em microambientes nem sempre é possível.

\section{MICROELETRODOS DE DIFERENTES GEOMETRIAS FABRICADOS POR MICROFABRICAÇÃO}

Microeletrodos com geometrias bem definidas (disco, banda, cônico, hemisférico, anel e esférico) e com alta eficiência de aproveitamento no processo de construção podem ser fabricados utilizando técnicas de microfabricação. ${ }^{38,95,125-127}$ O processo de litografia, utilizado na maioria dos casos de microfabricação, está baseado na transferência de uma cópia de um padrão mestre, de um determinado material, para um substrato sólido. Um exemplo é a fabricação de microeletrodos cônicos a partir do desgaste de um substrato de Si, seguido pela deposição de filmes finos de $\mathrm{SiO}_{2}, \mathrm{Pt}$ e $\mathrm{Si}_{3} \mathrm{~N}_{4}$. Em seguida, uma camada sensível à radiação ultravioleta é depositada, deixando somente a ponta do microeletrodo descoberta. O filme de $\mathrm{Si}_{3} \mathrm{~N}_{4}$ que recobre a ponta cônica do microeletrodo de Pt é então removido usando desgaste por plasma. Esse processo permite a construção de microeletrodos com $1,2 \mu \mathrm{m}$ de raio. ${ }^{128}$

\section{MICROELETRODOS DE PORO EM VIDRO}

Microeletrodos de poro em vidro ${ }^{70,129-131}$ podem ser fabricados a partir do desgaste eletroquímico de fibras metálicas encapsuladas em vidro. O processo para fabricação desse tipo de microeletrodo está mostrado na Figura 8, sendo que o raio do poro pode variar de $5 \mathrm{~nm}$ a 1 $\mu \mathrm{m}$. Esse tipo de eletrodo, cuja construção é simples e não requer o uso de equipamentos sofisticados, possibilita a investigação do transporte de moléculas através de orifícios de dimensões nanométricas. Além disso, pode propiciar maior seletividade ${ }^{132,133}$ aos métodos eletroanalíticos, pois o tamanho do poro e eventuais processos de modificação da superfície do vidro podem limitar a interferência causada por compostos de tamanho maior que o do poro. Vantagens adicionais são associadas ao uso de eletrodos com poros cônicos em relação aos de poros cilín- 
dricos como, por exemplo, alta condutância iônica, possibilidade de estudos em meios com alta resistência sem haver problemas de queda ôhmica ${ }^{131}$ e independência do fluxo de moléculas no estado estacionário em relação à profundidade do poro. ${ }^{130}$

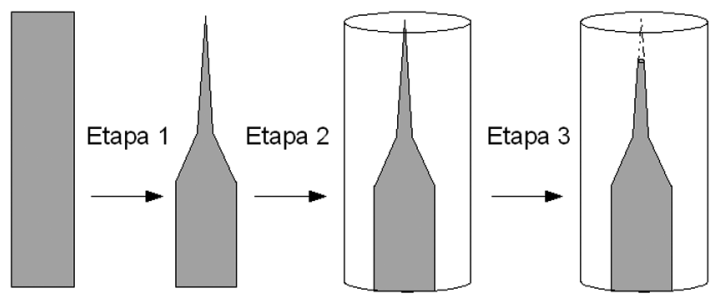

Figura 8. Esquema do processo de fabricação de um microeletrodo de poro em vidro. Etapa 1: desgaste eletroquímico da microfibra de platina; etapa 2: processo de selagem da microfibra no vidro e etapa 3: desgaste eletroquímico da microfibra encapsulad $a^{70}$

\section{CONJUNTO E ARRANJO DE MICROELETRODOS}

Além da dificuldade de fabricação, microeletrodos individuais de pequenas dimensões apresentam uma desvantagem adicional relacionada à necessidade de monitoramento de correntes muito baixas, às vezes na casa de fA. Entretanto, ao se trabalhar com ensembles (conjuntos) e arrays (arranjos) de microeletrodos individuais, correntes significativamente mais altas podem ser obtidas se os dispositivos individuais forem interconectados. Há diversas estratégias para a confecção de conjunto ou arranjo de microeletrodos de diferentes geometrias e a denominação (arranjo ou conjunto) dependerá do controle (arranjo = ordenado) ou não (conjunto = aleatório) da distância entre os microeletrodos. Nestes casos, a distância entre os microeletrodos individuais constitui-se em aspecto importante, pois cálculos teóricos prevêem que para microeletrodos de disco de raio $a$ montados de acordo com a configuração de arranjo, a interpenetração entre as camadas de difusão dos microeletrodos individuais ocorre desde que a distância entre os microeletrodos seja inferior a $6 a^{25}$. Caso a interpenetração das camadas de difusão prevaleça, voltamogramas convencionais com os componentes anódicos e catódicos serão obtidos.

Diversos métodos são reportados na literatura para a fabricação de conjunto e arranjo de microeletrodos, sendo alguns deles derivados da construção de microeletrodos individuais. Dentre esses métodos podem ser citados aqueles envolvendo técnicas de microfabricação, ${ }^{16,125,134-136}$ métodos mecânicos com fios ${ }^{25,137-141}$ e sanduíches de filmes finos de camadas metálicas, ${ }^{118,142,143}$, formação de compósitos, ${ }^{144,145}$ sonoquímica, ${ }^{146-148}$ utilização de moldes preparados por nanomateriais ${ }^{12,149-158}$ e de líquidos iônicos ${ }^{159-161}$ e máscaras de toner. ${ }^{162}$ Procedimento simples, engenhoso e barato foi desenvolvido por Angnes e colaboradores ${ }^{163}$ e fundamenta-se na obtenção de microdiscos de ouro a partir do corte transversal em chips comerciais e posterior etapa de polimento até o aparecimento das microfibras.

\section{CONCLUSÕES E PERSPECTIVAS}

Diferentes métodos para a fabricação de microeletrodos e conjunto/arranjo de microeletrodos foram descritos nesse artigo. A construção de sensores descartáveis, de baixo custo e que possuam estas características consiste em campo de pesquisa atrativo e aberto para novas metodologias. Um dos desafios a serem enfrentados está pautado no controle e na alta reprodutibilidade da área dos microeletrodos, visando processos de construção em larga escala.

A construção de dispositivos cada vez menores, com tamanho total em escala submicrométrica, constitui-se em outra meta para pesquisadores que desenvolvem trabalhos nesta área. Microeletrodos estruturalmente microscópicos ou "nanoscópicos" podem ser empregados como poderosa ferramenta para a análise em microambientes e em tempo real. Neste contexto, determinações efetuadas com volumes reduzidos (nL) são bastante importantes no caso de amostras dispendiosas ou de fluidos biológicos (por exemplo, sangue). O uso destes microssensores em sistemas biológicos também é atrativo, pois o mecanismo de funcionamento das células pode ser investigado in situ, em tempo real e sem destruição da amostra. A elevada resolução espacial e o rápido tempo de resposta dos microeletrodos propiciam a obtenção de mapas bidimensionais de ambientes vizinhos à célula, os quais podem contribuir para elucidar o transporte de compostos entre neurônios e membranas celulares. O desenvolvimento de arranjos de microeletrodos com propriedades específicas de reconhecimento e a utilização na captura dessas informações de maneira simultânea certamente será interessante para pesquisadores da área biológica.

A possibilidade de investigar superfícies empregando-se técnicas como a microscopia eletrônica de varredura e a microscopia de força atômica ampliou de maneira significativa a compreensão de fenômenos em escala molecular. Estas técnicas possuem elevada resolução espacial, mas pouca informação sobre especificidade química é obtida. Neste contexto, cabe ressaltar o emprego de microeletrodos como efetivos sensores de reatividade em microscopia eletroquímica. Esta técnica, apesar da menor resolução espacial, preenche esta lacuna visto que existe interação eletroquímica da espécie ou superfície investigada com o microssensor.

Finalmente, cumpre destacar outra área que vem crescendo de maneira significativa nos últimos anos em Química Analítica. Trata-se do desenvolvimento de sistemas que realizam de maneira integrada todas as etapas do método analítico (desde a preparação da amostra até a detecção), os chamados $\mu$ TAS (micro total analysis system) ou lab-on-a-chip. Devido às propriedades dos microeletrodos, principalmente o tamanho, e à possibilidade de miniaturização dos potenciostatos, sensores de dimensões microscópicas são altamente desejáveis, pois podem atuar como detectores nestes microssistemas de análise. Abrem-se, desta maneira, novas possibilidades e perspectivas para a fabricação de microeletrodos de configurações apropriadas para a inserção nestes instrumentos.

Em resumo, microeletrodos estimulam pesquisas de cunho interdisciplinar, pois os desafios a serem suplantados exigem soluções criativas resultantes da interação entre pesquisadores de áreas distintas. A capacidade destes dispositivos em explorar eventos químicos em domínios microscópicos os credencia como ferramenta valiosa para estudos em sistemas de interesse biológico, em que o transporte de íons e moléculas é fundamental para a garantia de uma condição estável. Evidencia-se, desta maneira, a existência de um fértil leque de aplicações dos microeletrodos, muitas das quais podem ser transformadas em conhecimento de fronteira e com perspectivas de largo impacto na comunidade científica.

\section{AGRADECIMENTOS}

À FAPESP, CAPES e ao CNPq pelo apoio financeiro concedido na forma de bolsas e para o financiamento das pesquisas.

\section{REFERÊNCIAS}

1. Stulik, K.; Amatore, C.; Holub, K.; Marecek, V.; Kutner, W.; Pure Appl. Chem. 2000, 72, 1483.

2. Adams, R. N.; Anal. Chem. 1976, 48, 1126.

3. Wightman, R. M.; Anal. Chem. 1981, 53, 1125.

4. Howell, J. O.; Wightman, R. M.; J. Phys. Chem. 1984, 88, 3915.

5. Howell, J. O.; Wightman, R. M.; Anal. Chem. 1984, 56, 524.

6. Wipf, D. O.; Michael, A. C.; Wightman, R. M.; J. Electroanal. Chem. 1989, 269, 15. 
7. Wightman, R. M.; Wipf, D. O.; Electroanal Chem. 1989, 15, 267.

8. Bond, A. M.; Fleischmann, M.; Robinson, J.; J. Electroanal. Chem. 1984, 180, 257.

9. Bond, A. M.; Fleischmann, M.; Robinson, J.; J. Electroanal. Chem. 1984, 168, 299.

10. Correia, A. N.; Mascaro, L. H.; Machado, S. A. S.; Mazo, L. H.; Avaca, L. A.; Quim. Nova 1995, 18, 475.

11. Silva, S. M.; Alves, C. R.; Correia, A. N.; Martins, R. M.; Nobre, A. L. R.; Machado, S. A. S.; Mazo, L. H.; Avaca, L. A.; Quim. Nova 1998, 21,78 .

12. Pereira, F. C.; Bergamo, E. P.; Zanoni, M. V. B.; Moretto, L. M.; Ugo, P.; Quim. Nova 2006, 29, 1054.

13. Ordeig, O.; del Campo, J.; Munoz, F. X.; Banks, C. E.; Compton, R. G.; Electroanalysis 2007, 19, 1973.

14. Shi, H. B.; Yeh, J. I.; Nanomedicine 2007, 2, 587.

15. Bond, A. M.; Analyst 1994, 119, R1.

16. Feeney, R.; Kounaves, S. P.; Electroanalysis 2000, 12, 677.

17. Wightman, R. M.; Science 2006, 311, 1570.

18. Wightman, R. M.; Science 1988, 240, 415.

19. Wightman, R. M.; Wipf, D. O. Em Electroanalytical Chemistry; Bard, A. J., ed.; Marcel Dekker: New York, 1989, vol. 15.

20. Fan, F. R. F.; Demaille, C. Em Scanning Electrochemical Microscopy; Bard, A. J.; Mirkin, C. A., eds.; Marcel Dekker: New York, 2001, cap. 3.

21. Bard, A. J.; Fan, F. R. F.; Mirkin, C. A. Em Electroanalytical Chemistry; Bard, A. J., ed.; Marcel Dekker: New York, 1994, vol. 18.

22. Powell, P. R.; Woods, L. A.; Ewing, A. G.; J. Sep. Sci. 2005, $28,2540$.

23. Wang, C. Y.; Chen, Y. J.; Wang, F. X.; Hu, X. Y.; Electrochim. Acta 2005, $50,5588$.

24. Hermans, A.; Wightman, R. M.; Langmuir 2006, 22, 10348.

25. Caudill, W. L.; Howell, J. O.; Wightman, R. M.; Anal. Chem. 1982, 54, 2532.

26. Wehmeyer, K. R.; Wightman, R. M.; Anal. Chem. 1985, 57, 1989.

27. Baer, C. D.; Stone, N. J.; Sweigart, D. A.; Anal. Chem. 1988, 60, 188.

28. Fernandez, A. I. S.; Calzon, J. A. G.; Garcia, A. C.; Blanco, P. T.; Electroanalysis 1991, 3, 413.

29. Bond, A. M.; Luscombe, D.; Oldham, K. B.; Zoski, C. G.; J. Electroanal. Chem. 1988, 249, 1.

30. Fan, F. R. F.; Fernandez, J.; Liu, B.; Mauzeroll, J.; Zoski, C. G. Em Handbook of Electrochemistry; Zoski, C. G., ed.; Elsevier: Boston, 2007, cap. 6.

31. Pendley, B. D.; Abruna, H. D.; Anal. Chem. 1990, 62, 782.

32. Shao, Y. H.; Mirkin, M. V.; Fish, G.; Kokotov, S.; Palanker, D.; Lewis, A.; Anal. Chem. 1997, 69, 1627.

33. Katemann, B. B.; Schuhmann, T.; Electroanalysis 2002, 14, 22.

34. Seddon, B. J.; Shao, Y.; Fost, J.; Girault, H. H.; Electrochim. Acta 1994, 39,783 .

35. Zhao, G.; Giolando, D. M.; Kirchhoff, J. R.; J. Electroanal. Chem. 1994, 379, 505.

36. Zhao, G.; Giolando, D. M.; Kirchhoff, J. R.; Anal. Chem. 1995, 67, 1491.

37. Zhao, G.; Giolando, D. M.; Kirchhoff, J. R.; Anal. Chem. 1995, 67, 2592.

38. Xia, Y. N.; Rogers, J. A.; Paul, K. E.; Whitesides, G. M.; Chem. Rev. 1999, 99, 1823.

39. Bozon, J. P.; Giolando, D. M.; Kirchhoff, J. R.; Electroanalysis 2001, $13,911$.

40. Giolando, D. M.; Kirchhoff, J. R.; Mueller, H.; Nguyen, P. Q.; Odeh, I. N.; Chem. Vapor Depos. 2002, 8, 93.

41. Zhu, M. Z.; Jiang, Z. D.; Jing, W. X.; Chem. J. Chin. Univ.-Chin. 2006, $27,233$.

42. Kaempgen, M.; Roth, S.; Synth. Met. 2005, 152, 353.

43. Miles, D. T.; Knedlik, A.; Wipf, D. O.; Anal. Chem. 1997, 69, 1240.

44. El-Giar, E.; Wipf, D. O.; J. Electroanal. Chem. 2007, 609, 147.

45. Li, C. Y.; Xu, X. Z.; Tigwell, D.; J. Neurosci. Methods 1995, 57, 217.
46. Lu, X.; Huang, W. H.; Wang, Z. L.; Cheng, H. K.; Anal. Chim. Acta 2004, 510, 127

47. Schulte, A.; Schuhmann, W.; Angew. Chem., Int. Edit. 2007, 46, 8760.

48. Wilson, G. S.; Gifford, R.; Biosens. Bioelectron. 2005, 20, 2388.

49. Wightman, R. M.; Runnels, P.; Troyer, K.; Anal. Chim. Acta 1999, 400, 5.

50. Cannon, D. M.; Winograd, N.; Ewing, A. G.; Annu. Rev. Biophys. Biomolec. Struct. 2000, 29, 239.

51. Yotter, R. A.; Wilson, D. M.; IEEE Sens. J. 2004, 4, 412.

52. Paixão, T. R. L. C.; Barbosa, L. F.; Carrì, M. T.; Medeiros, M. H. G.; Bertotti, M.; Analyst 2008, 133, 1605.

53. Strein, T. G.; Ewing, A. G.; Anal. Chem. 1992, 64, 1368.

54. Malinski, T.; Taha, Z.; Nature 1992, 358, 676.

55. Huang, W. H.; Pang, D. W.; Tong, H.; Wang, Z. L.; Cheng, J. K.; Anal. Chem. 2001, 73, 1048.

56. Zhang, X. J.; Zhang, W. M.; Zhou, X. Y.; Ogorevc, B.; Anal. Chem. 1996, 68, 3338.

57. Penner, R. M.; Heben, M. J.; Longin, T. L.; Lewis, N. S.; Science 1990, 250,1118

58. Nagahara, L. A.; Thundat, T.; Lindsay, S. M.; Rev. Sci. Instrum. 1989, 60,3128 .

59. Kawagoe, K. T.; Jankowski, J. A.; Wightman, R. M.; Anal. Chem. 1991, $63,1589$.

60. Chen, S. L.; Kucernak, A.; J. Phys. Chem. B 2002, 106, 9396.

61. Chen, S. L.; Kucernak, A.; Electrochem. Commun. 2002, 4, 80.

62. Kuras, A.; Gutmaniene, N.; J. Neurosci. Methods 2000, 96, 143.

63. Slevin, C. J.; Gray, N. J.; Macpherson, J. V.; Webb, M. A.; Unwin, P. R.; Electrochem. Commun. 1999, 1, 282.

64. Mirkin, M. V.; Fan, F. R. F.; Bard, A. J.; J. Electroanal. Chem. 1992, $328,47$.

65. Watkins, J. J.; Chen, J. Y.; White, H. S.; Abruna, H. D.; Maisonhaute, E.; Amatore, C.; Anal. Chem. 2003, 75, 3962.

66. Sun, P.; Zhang, Z. Q.; Guo, J. D.; Shao, Y. H.; Anal. Chem. 2001, 73, 5346.

67. Conyers, J. L.; White, H. S.; Anal. Chem. 2000, 72, 4441.

68. Gray, N. J.; Unwin, P. R.; Analyst 2000, 125, 889.

69. Woo, D. H.; Kang, H.; Park, S. M.; Anal. Chem. 2003, 75, 6732

70. Zhang, B.; Galusha, J.; Shiozawa, P. G.; Wang, G. L.; Bergren, A. J.; Jones, R. M.; White, R. J.; Ervin, E. N.; Cauley, C. C.; White, H. S.; Anal. Chem. 2007, 79, 4778.

71. Sorensen, A. H.; Hvid, U.; Mortensen, M. W.; Morch, K. A.; Rev. Sci. Instrum. 1999, 70, 3059.

72. Libioulle, L.; Houbion, Y.; Gilles, J. M.; Rev. Sci. Instrum. 1995, 66, 97.

73. Song, J. P.; Pryds, N. H.; Glejbol, K.; Morch, K. A.; Tholen, A. R.; Christensen, L. N.; Rev. Sci. Instrum. 1993, 64, 900.

74. Paixão, T. R. L. C.; Lowinsohn, D.; Bertotti, M.; J. Agric. Food Chem. 2006, 54, 3072 .

75. Abe, T.; Itaya, K.; Uchida, I.; Chem. Lett. 1988, 399.

76. Gewirth, A. A.; Craston, D. H.; Bard, A. J.; J. Electroanal. Chem. 1989, $261,477$.

77. Vitus, C. M.; Chang, S. C.; Schardt, B. C.; Weaver, M. J.; J. Phys. Chem. 1991, 95, 7559

78. Zhang, B. L.; Wang, E. K.; Electrochim. Acta 1994, 39, 103.

79. Penner, R. M.; Heben, M. J.; Lewis, N. S.; Anal. Chem. 1989, 61, 1630.

80. Lee, Y.; Amemiya, S.; Bard, A. J.; Anal. Chem. 2001, 73, 2261.

81. Lee, Y.; Bard, A. J.; Anal. Chem. 2002, 74, 3626.

82. Macpherson, J. V.; Unwin, P. R.; Anal. Chem. 2000, 72, 276

83. Bach, C. E.; Nichols, R. J.; Meyer, H.; Besenhard, J. O.; Surf. Coat. Technol. 1994, 67, 139

84. Bach, C. E.; Nichols, R. J.; Beckmann, W.; Meyer, H.; Schulte, A.; Besenhard, J. O.; Jannakoudakis, P. D.; J. Electrochem. Soc. 1993, 140, 1281.

85. Mao, B. W.; Ye, J. H.; Zhuo, X. D.; Mu, J. Q.; Fen, Z. D.; Tian, Z. W.; Ultramicroscopy 1992, 42, 464 
86. Schulte, A.; Chow, R. H.; Anal. Chem. 1996, 68, 3054.

87. El-Giar, E. E. M.; Wipf, D. O.; Electroanalysis 2006, 18, 2281.

88. Kuhn, L. S.; Weber, A.; Weber, S. G.; Anal. Chem. 1990, 62, 1631.

89. Pennarun, G. I.; Boxall, C.; Ohare, D.; Analyst 1996, 121, 1779.

90. Casillas, N.; James, P.; Smyrl, W. H.; J. Electrochem. Soc. 1995, 142, L16.

91. James, P.; Casillas, N.; Smyrl, W. H.; J. Electrochem. Soc. 1996, 143, 3853.

92. Strein, T. G.; J. Electrochem. Soc. 1991, 138, C254.

93. Paixão, T. R. L. C.; Cardoso, J. L.; Bertotti, M.; Fuel 2007, 86, 1185.

94. Lee, C.; Miller, C. J.; Bard, A. J.; Anal. Chem. 1991, 63, 78.

95. Kranz, C.; Friedbacher, G.; Mizaikoff, B.; Anal. Chem. 2001, 73, 2491.

96. Kim, Y. T.; Scarnulis, D. M.; Ewing, A. G.; Anal. Chem. 1986, 58, 1782.

97. Saraceno, R. A.; Ewing, A. G.; Anal. Chem. 1988, 60, 2016.

98. Cohen, C. B.; Weber, S. G.; Anal. Chem. 1993, 65, 169.

99. Betzig, E.; Trautman, J. K.; Science 1992, 257, 189.

100. Lee, Y.; Ding, Z. F.; Bard, A. J.; Anal. Chem. 2002, 74, 3634

101. Demaille, C.; Brust, M.; Tsionsky, M.; Bard, A. J.; Anal. Chem. 1997, 69, 2323.

102. Soos, Z. G.; Lingane, P. J.; J. Phys. Chem. 1964, 68, 3821

103. Newman, J.; J. Electrochem. Soc. 1966, 113, 501.

104. Pons, S.; Fleischmann, M.; Anal. Chem. 1987, 59, A1391.

105. Flanagan, J. B.; Marcoux, L.; J. Phys. Chem. 1973, 77, 1051.

106. Dayton, M. A.; Brown, J. C.; Stutts, K. J.; Wightman, R. M.; Anal. Chem. 1980, 52, 946.

107. Oldham, K. B.; J. Electroanal. Chem. 1981, 122, 1.

108. Galus, Z.; Schenk, J. O.; Adams, R. N.; J. Electroanal. Chem. 1982, $135,1$.

109. Aoki, K.; Osteryoung, J.; J. Electroanal. Chem. 1981, 122, 19

110. Aoki, K.; Osteryoung, J.; J. Electroanal. Chem. 1981, 125, 315.

111. Aoki, K.; Akimoto, K.; Tokuda, K.; Matsuda, H.; Osteryoung, J.; J. Electroanal. Chem. 1984, 171, 219.

112. Shoup, D.; Szabo, A.; J. Electroanal. Chem. 1982, 140, 237.

113. Mauzeroll, J.; Hueske, E. A.; Bard, A. J.; Anal. Chem. 2003, 75, 3880.

114. Kowalski, Z.; Wong, K. H.; Osteryoung, R. A.; Osteryoung, J.; Anal. Chem. 1987, 59, 2216.

115. Stojek, Z.; Osteryoung, J.; Anal. Chem. 1988, 60, 131.

116. Morris, R. B.; Franta, D. J.; White, H. S.; J. Phys. Chem. 1987, 91, 3559.

117. Kovach, P. M.; Caudill, W. L.; Peters, D. G.; Wightman, R. M.; J. Electroanal. Chem. 1985, 185, 285.

118. Welford, P. J.; Freeman, J.; Wilkins, S. J.; Wadhawan, J. D.; Hahn, C. E. W.; Compton, R. G.; Anal. Chem. 2001, 73, 6088.

119. Craston, D. H.; Jones, C. P.; Williams, D. E.; Elmurr, N.; Talanta 1991, $38,17$.

120. Chang, J. L.; Zen, J. M.; Electrochem. Commun. 2006, 8, 571.

121. Rawson, F. J.; Purcell, W. M.; Xu, J.; Cowell, D. C.; Fielden, P. R.; Biddle, N.; Hart, J. P.; Electrochim. Acta 2007, 52, 7248.

122. Samuelsson, M.; Armgarth, M.; Nylander, C.; Anal. Chem. 1991, 63, 931.

123. Seddon, B. J.; Eddowes, M. J.; Firth, A.; Owen, A. E.; Girault, H. H. J.; Electrochim. Acta 1991, 36, 763

124. Chang, J. L.; Zen, J. M.; Electroanalysis 2006, 18, 941.

125. Feeney, R.; Herdan, J.; Nolan, M. A.; Tan, S. H.; Tarasov, V. V.; Kounaves, S. P.; Electroanalysis 1998, 10, 89.

126. Henry, C. S.; Fritsch, I.; J. Electrochem. Soc. 1999, 146, 3367.

127. Henry, C. S.; Fritsch, I.; Anal. Chem. 1999, 71, 550.

128. Thiebaud, P.; Beuret, C.; de Rooij, N. F.; Koudelka-Hep, M.; Sens. Actuator B-Chem. 2000, 70, 51.
129. Zhang, B.; Zhang, Y. H.; White, H. S.; Anal. Chem. 2004, 76, 6229.

130. Zhang, B.; Zhang, Y. H.; White, H. S.; Anal. Chem. 2006, 78, 477.

131. Zhang, Y. H.; Zhang, B.; White, H. S.; J. Phys. Chem. B 2006, 110, 1768 .

132. Lee, S.; Zhang, Y. H.; White, H. S.; Harrell, C. C.; Martin, C. R.; Anal. Chem. 2004, 76, 6108

133. Heins, E. A.; Siwy, Z. S.; Baker, L. A.; Martin, C. R.; Nano Lett. 2005, 5,1824 .

134. Lowinsohn, D.; Peres, H. E. M.; Kosminsky, L.; Paixão, T. R. L. C.; Ferreira, T. L.; Ramirez-Fernandez, F. J.; Bertotti, M.; Sens. Actuator B-Chem. 2006, 113, 80.

135. Daniele, S.; De Faveri, E.; Kleps, I.; Angelescu, A.; Electroanalysis 2006, 18,1749

136. Beni, V.; Arrigan, D. W. M.; Curr. Anal. Chem. 2008, 4, 229.

137. Nagale, M. P.; Fritsch, I.; Anal. Chem. 1998, 70, 2902.

138. Connolly, P.; Moores, G. R.; Monaghan, W.; Shen, J.; Britland, S.; Clark, P.; Sens. Actuator B-Chem. 1992, 6, 113.

139. Nagale, M. P.; Fritsch, I.; Anal. Chem. 1998, 70, 2908.

140. Schwarz, J.; Kaden, H.; Enseleit, U.; Electrochem. Commun. 2000, 2 , 606.

141. Xu, X.; Liu, C.; Jia, J.; Liu, B.; Yang, X.; Dong, S.; Electroanalysis 2008, 20, 797.

142. Fosset, B.; Amatore, C.; Bartelt, J.; Wightman, R. M.; Anal. Chem. 1991, 63, 1403.

143. Fosset, B.; Amatore, C. A.; Bartelt, J. E.; Michael, A. C.; Wightman, R. M.; Anal. Chem. 1991, 63, 306.

144. Weisshaar, D. E.; Tallman, D. E.; Anal. Chem. 1983, 55, 1146

145. Wang, J.; Angnes, L.; Tobias, H.; Roesner, R. A.; Hong, K. C.; Glass, R. S.; Kong, F. M.; Pekala, R. W.; Anal. Chem. 1993, 65, 2300.

146. Barton, A. C.; Collyer, S. D.; Davis, F.; Gornall, D. D.; Law, K. A.; Lawrence, E. C. D.; Mills, D. W.; Myler, S.; Pritchard, J. A.; Thompson, M.; Higson, S. P. J.; Biosens. Bioelectron. 2004, 20, 328.

147. Davis, F.; Collyer, S. D.; Gornall, D. D.; Law, K. A.; Mills, D. W.; Higson, S.; Chim. Oggi-Chem. Today 2007, 25, 28.

148. Simm, A. O.; Ward-Jones, S.; Banks, C. E.; Compton, R. G.; Anal. Sci. 2005, 21, 667.

149. Yang, M. H.; Qu, F. L.; Lu, Y. S.; He, Y.; Shen, G. L.; Yu, R. Q.; Biomaterials 2006, 27, 5944.

150. Martin, C. R.; Science 1994, 266, 1961.

151. Penner, R. M.; Martin, C. R.; Anal. Chem. 1987, 59, 2625

152. Merritt, C. D.; Justus, B. L.; Chem. Mat. 2003, 15, 2520.

153. Szunerits, S.; Walt, D. R.; Anal. Chem. 2002, 74, 1718.

154. Szunerits, S.; Tam, J. M.; Thouin, L.; Amatore, C.; Walt, D. R.; Anal. Chem. 2003, 75, 4382

155. Wang, C. Y.; Shao, X. Q.; Liu, Q. X.; Mao, Y. D.; Yang, G. J.; Xue, H. G.; Hu, X. Y.; Electrochim. Acta 2006, 52, 704.

156. Wang, C. Y.; Liu, Q. X.; Shao, X. Q.; Yang, G. J.; Xue, H. G.; Hu, X. Y.; Talanta 2007, 71, 178.

157. Ito, T.; Audi, A. A.; Dible, G. P.; Anal. Chem. 2006, 78, 7048.

158. Krishnamoorthy, K.; Zoski, C. G.; Anal. Chem. 2005, 77, 5068.

159. Elliott, J. M.; Birkin, P. R.; Bartlett, P. N.; Attard, G. S.; Langmuir 1999, $15,7411$.

160. Daniele, S.; Bergamin, S.; Electrochem. Commun. 2007, 9, 1388

161. Nie, M. Y.; Elliott, J. M.; J. Mater. Sci. Technol. 2005, 21, 863.

162. Richter, E. M.; Munoz, R. A. A.; Bertotti, M.; Angnes, L.; Electrochem. Commun. 2007, 9, 1091

163. Augelli, M. A.; Nascimento, V. B.; Pedrotti, J. J.; Gutz, I. G. R.; Angnes, L.; Analyst 1997, 122, 843 